\title{
Modulation of a cAMP/Protein Kinase A Cascade by Protein Kinase C in Sensory Neurons of Aplysia
}

\author{
Shuzo Sugita, Douglas A. Baxter, and John H. Byrne \\ Department of Neurobiology and Anatomy, The University of Texas Medical School at Houston, Houston, Texas 77225
}

The synaptic connections between the sensory neurons of Aplysia and their follower neurons have been used as a model system for examining the cellular mechanisms contributing to neuronal and synaptic plasticity. Recent studies suggest that at least two protein kinases, protein kinase $A(P K A)$ and protein kinase $\mathrm{C}(\mathrm{PKC})$, contribute to serotonin (5-HT)-induced shortterm facilitation. The interaction between these two kinase cascades has not been examined, however. Using electrophysiological and biochemical approaches, we examined possible interactions between PKA and PKC cascades. The results indicated that prolonged activation of PKC by preincubation with phorbol esters attenuated PKA-mediated actions of 5-HT, including increases in sensory neuron excitability and spike broadening in the presence of tetraethylammonium (TEA) and nifedipine. Although phorbol esters also attenuated increases in excitability by an analog of cAMP and small cardioactive pep- tide $\mathrm{B}\left(\mathrm{SCP}_{\mathrm{B}}\right)$, the degree of attenuation was smaller. In addition, phorbol esters did not attenuate broadening of TEA spikes by the cAMP analog and $\mathrm{SCP}_{\mathrm{B}}$. Thus, phorbol esters appeared specifically to attenuate aspects of the 5- HT activation of the cAMP/PKA cascade. Measurements of CAMP levels with radioimmunoassays revealed that phorbol esters did not attenuate 5-HT-induced cAMP synthesis, however. Finally, the results indicated that phorbol esters themselves induced a small but significant increase in excitability as well as an increase in the level of cAMP. Our results suggest that there is crosstalk between the PKC and PKA cascades. The mechanisms by which phorbol esters specifically attenuate 5-HT-induced activation of the cAMP/PKA cascade are not known, however.

Key words: Aplysia californica; protein kinase A; protein kinase $C$; cyclic AMP; crosstalk; synaptic facilitation; modulation; serotonin; sensory neurons; learning and memory
Serotonergic modulation of sensory neurons in Aplysia has been used extensively as a model system with which to study neuronal plasticity (Kandel and Schwartz, 1982; Carew and Sahley, 1986; Byrne, 1987; Hawkins et al., 1993; Byrne and Kandel, 1996). At least two protein kinases, protein kinase A (PKA) and protein kinase $\mathrm{C}$ (PKC), mediate the serotonin (5-HT)-induced shortterm facilitation, and the actions of these two kinase cascades are time- and state-dependent (Byrne and Kandel, 1996). Possible interactions or crosstalk between these two kinase cascades has not been examined, however. Several lines of evidence suggest that some crosstalk may occur. First, Wu et al. (1994) found that H-7, a selective inhibitor of PKC in sensory neurons of Aplysia, blocked 5-HT-induced long-term facilitation, which is believed to be mediated primarily by the PKA cascade (Schacher et al., 1988, 1993; Scholz and Byrne; 1988; Dash et al., 1990; Nazif et al., 1991; Ghirardi et al., 1995; O'Leary et al., 1995). Second, an interaction between the PKA and PKC cascades in the sensory neurons of Aplysia was suggested by Sugita et al. (1992). They found that activators of PKC caused an increase in excitability, a well known cAMP-dependent effect (Klein et al., 1986; Baxter and Byrne, 1989). Furthermore, the addition of 5-HT to the bath that already contained the PKC activator did not increase excitability as much

Received June 30, 1997; accepted July 14, 1997.

This work was supported by National Institute of Mental Health Award K05 MH-00649 and National Institutes of Health Research Grant R01 NS-19895. We thank Kyoko Sugita for help with the illustrations.

Correspondence should be addressed to Dr. John H. Byrne, Department of Neurobiology and Anatomy, The University of Texas Medical School at Houston, P.O. Box 20708, Houston, TX 77225.

Dr. Sugita's present address: Department of Molecular Genetics, University of Texas Southwestern Medical Center at Dallas, 5323 Harry Hines Boulevard, Dallas, TX 75235-9046.

Copyright (C) 1997 Society for Neuroscience $\quad 0270-6474 / 97 / 177237-08 \$ 05.00 / 0$ as the application of 5-HT alone. Similar interactions were observed in spike broadening and synaptic facilitation (Sugita et al., 1992, 1997), leading to the hypothesis that activation of PKC may modulate, or interact with, the cAMP/PKA cascade.

In other cellular systems crosstalk between PKA and PKC cascades has been suggested. One locus of interaction is the G-protein $\left(\mathrm{G}_{\mathrm{s}}\right)$-sensitive adenylyl cyclase. PKC increases the activity of adenylyl cyclases and the level of cAMP in intact cells and cell-free systems (Pieroni et al., 1993; Cooper et al., 1995). PKC also is known to phosphorylate various types of the receptors for ligands (Huganir and Greengard, 1990). Phosphorylation of the receptors normally results in their desensitization and an inhibition of the ligand-induced increases in intracellular messengers, including cAMP.

Using electrophysiological and biochemical approaches, we examined the interaction between the two cascades (PKA and PKC pathways), in particular the effects of PKC on the cAMP/PKA pathway. Results are presented from three complementary measurements of cAMP and its actions in sensory neurons, including (1) measurements of increased excitability, (2) measurements of spike broadening in the presence of tetraethylammonium (TEA) and nifedipine, and (3) measurements of the intracellular levels of cAMP. In addition, we compared results among several different pharmacological manipulations [e.g., application of cAMP ana$\log$, 5-HT, or small cardioactive peptide $\left.\mathrm{B}\left(\mathrm{SCP}_{\mathrm{B}}\right)\right]$ that can increase cAMP levels. The results indicated that activation of $\mathrm{PKC}$ by phorbol esters specifically attenuated aspects of the 5-HT activation of the $\mathrm{cAMP} / \mathrm{PKA}$ cascade, although phorbol esters did not attenuate 5-HT-induced cAMP synthesis. In addition, phorbol esters themselves induced a small but significant increase in excitability as well as an increase in the level of cAMP. Our 
results suggest that there is crosstalk between PKC and PKA cascades.

\section{MATERIALS AND METHODS}

Chemicals. Serotonin creatine sulfate (5-HT; Sigma, St. Louis, MO), 8-bromo-cAMP (Sigma), and TEA (Kodak, Rochester, NY) were dissolved in artificial seawater (ASW; Instant Ocean) and prepared daily. Stock solutions of nifedipine (20 mM; Sigma) were dissolved in DMSO (Sigma) and prepared daily. Stock solutions of $\mathrm{SCP}_{\mathrm{B}}(10 \mathrm{~mm}$ in distilled water; Peninsula, Belmont, CA) were stored at $-20^{\circ} \mathrm{C}$. Two types of phorbol esters, $4 \beta$-phorbol 12,13-diacetate (PDAc; Sigma) and $4 \beta-12$ deoxyphorbol 13-isobutyrate (DPB; LC Laboratories, Woburn, MA) were used to activate PKC. Inactive $4 \alpha$-phorbols (Sigma) also were used as control. Stock solutions of all phorbols (10 mM in DMSO) were stored at $-20^{\circ} \mathrm{C}$. Small aliquots of concentrated agents were applied directly to the recording chamber.

Measurements of excitability. The techniques were essentially identical to those used in Sugita et al. (1992). Clusters of sensory neuron somata were isolated surgically from pleural ganglia and were pinned to the floor of a recording chamber that contained buffered ASW, pH 7.6. The preparations were maintained at $15 \pm 1^{\circ} \mathrm{C}$. Intracellular recordings were made by using conventional two-electrode current-clamp techniques, and recordings were made from only a single neuron per cluster. The excitability of a cell was measured by counting the number of action potentials elicited by a $1 \mathrm{sec}, 2 \mathrm{nA}$ depolarizing current pulse. The excitability was measured every $3 \mathrm{~min}$, and the baseline was defined as the average number of spikes elicited during the first three excitability measurements (e.g., see Fig. 3). Only those cells for which the baseline excitability was between two and eight spikes were accepted for further study. In control solutions (i.e., ASW), the average baseline excitability of most sensory neurons fell within this range. As described below, pretreatment with PDAc occasionally increased the baseline excitability of some cells to more than eight spikes. These cells were not accepted to reduce the possibility that a ceiling effect might affect the interpretation of the results.

Measurements of spike duration of TEA spikes. Single microelectrodes were used to record the duration of action potentials in the presence of TEA $(100 \mathrm{mM})$ and nifedipine $(10 \mu \mathrm{M})$. To prevent eliciting trains of action potentials, we injected the negative current via a recording electrode, and we maintained the resting potential at approximately $-65 \mathrm{mV}$. A pulse of $10-15 \mathrm{nA}$ (for $1.5 \mathrm{msec}$ ) was injected every 1 min to elicit an action potential. The duration of the TEA spikes was measured as the time between the peak of action potentials and $50 \%$ of the peak amplitude of the spike (Jarrard et al., 1993).

Analysis of electrophysiological data. Action potentials elicited in the sensory neuron were digitized on-line with a microcomputer and stored for later display and analysis. In each preparation data were normalized to the mean of three baseline measures of excitability or spike duration spikes before application of agents (i.e., the three measurements obtained immediately after the cell was penetrated with the microelectrode). Two-tailed statistics were used; $p$ values $<0.05$ were considered significant.

Measurements of cAMP concentrations in sensory neurons. Similar techniques were used as those described in Ocorr et al. (1986) and Sweatt et al. (1989). A cluster of $\sim 200$ sensory neuron somata was isolated surgically from a pleural ganglion by undercutting the somata with iridectomy scissors. Each isolated sensory cluster was equilibrated for $1 \mathrm{hr}$ in a microcentrifuge tube containing $40 \mu \mathrm{l}$ of ASW. (In some experimental groups ASW also contained $3 \mu \mathrm{M}$ PDAc or $\alpha$-phorbols.) In all experiments the isolated clusters then were exposed to the phosphodiesterase inhibitor RO 20-1724 (100 $\mu \mathrm{M}$; Biomol, Plymouth Meeting, PA) 3 min before the clusters were homogenized (see below). At $1 \mathrm{~min}$ after the application of RO 20-1724 (i.e., 2 min before the clusters were homogenized) either 5-HT (final concentration of $40 \mu \mathrm{M}$ ) or ASW was added to the tube.

The cAMP content of sensory neuron clusters was analyzed by radioimmunoassay, using a kit (Amersham, Arlington Heights, IL). At 2 min after exposure to experimental protocol (i.e., exposure to either $40 \mu \mathrm{M}$ 5-HT or ASW), ice-cold trichloroacetic acid (J. T. Baker, Phillipsburg, $\mathrm{NJ}$ ) was added to the solution (final concentration was $10 \% \mathrm{w} / \mathrm{v}$ ) containing the cluster of sensory neurons. Tissue was homogenized with a blue color pestle (Contes), and the supernatant was assayed for cAMP content. The protein content of both of the sensory neuron clusters was analyzed by bicinchoninic acid assays (Brown et al., 1989). Precipitated samples were added to $50 \mu \mathrm{l}$ of $5 \%(\mathrm{v} / \mathrm{v})$ SDS and $0.1 \mathrm{M} \mathrm{NaOH}$. After the
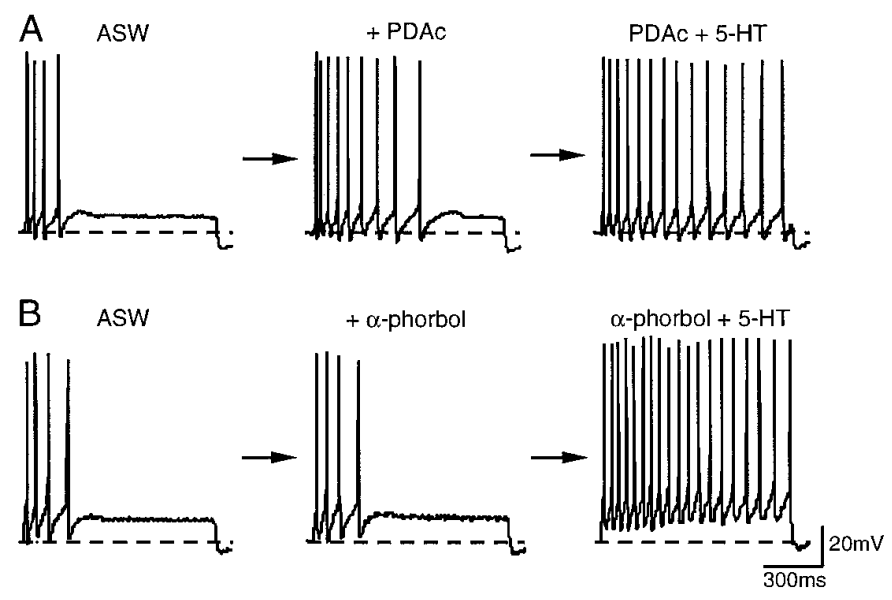

Figure 1. Interaction between PDAc- and 5-HT-induced enhancement of excitability. $A$, Excitability was measured before and $9 \mathrm{~min}$ after the application of PDAc $(3 \mu \mathrm{M})$ and 3 min after the addition of 5-HT $(10 \mu \mathrm{M})$ to the bath, which already contained PDAc, for $30 \mathrm{~min} . B$, Excitability was examined before and 9 min after the application of $\alpha$-phorbols $(3 \mu \mathrm{M})$ and 3 min after the application of 5-HT to the bath, which already contained $\alpha$-phorbols, for $30 \mathrm{~min}$.

addition of the reagents (Pierce, Rockford, IL), sample fluorescence was determined on a spectrofluorometer.

Because a single Aplysia has two symmetrical clusters of sensory neurons, each animal served as its own control, and a two-tailed $t$ test for nonindependent groups was used to analyze the data.

\section{RESULTS}

\section{Activation of PKC increased excitability and attenuated 5-HT-induced enhancement of excitability in the sensory neuron}

We first repeated the experiment reported in Sugita et al. (1992) and confirmed the results. In the present experiment we used two active derivatives of phorbol, DPB $(3 \mu \mathrm{M})$ and PDAc $(3 \mu \mathrm{M})$. Application of PDAc $(n=7)$ or DPB $(n=6)$ induced a gradual increase in excitability, which reached a peak in 9 min (at 9 min PDAc was $175 \pm 14 \%$ of baseline; DPB was $194 \pm 21 \%$ ) (Figs. $1 A, 2 A)$, whereas inactive phorbol ester, $\alpha$-phorbols $(3 \mu \mathrm{M} ; n=$ $6)$, had little effect $(123 \pm 9 \%)$ (Figs. $1 B, 2 A)$. A one-way ANOVA, which compared the effects of PDAc, DPB, and $\alpha$-phorbols on excitability, revealed that there was a significant difference among agents at 9 min after application $\left(F_{(2,16)}=5.55\right.$; $p<0.02)$. Post hoc analysis with Tukey tests indicated that there was a significant difference between DPB and $\alpha$-phorbol $\left(q_{(16,3)}=\right.$ 4.54; $p<0.02)$ and that there was no significant difference between PDAc and DPB $\left(q_{(16,3)}=1.29\right)$. Although in this series of experiments the difference between PDAc and $\alpha$-phorbols did not reach a significant level $\left(q_{(16,3)}=3.44 ; p=0.067\right)$, a previous experiment found a significant difference between the two (see Sugita et al., 1992). Thus, activators of PKC induced an increase in excitability.

The interaction between PKC- and 5-HT-induced enhancement of excitability was examined also. Serotonin $(10 \mu \mathrm{M})$ was added to the bath, which already contained one type of phorbol esters, for $27 \mathrm{~min}$ (DPB) or $30 \mathrm{~min}$ (PDAc and $\alpha$-phorbols). The 5-HT-induced enhancement of excitability was significantly attenuated by the preexposures to PDAc or DPB, when compared with the effect of 5-HT after $\alpha$-phorbols (3 min after application of 5-HT, PDAc was $269 \pm 28 \%$ of baseline; DPB was $248 \pm 42 \%$; $\alpha$-phorbols were $490 \pm 58 \%$ ) (Figs. 1, 2B). A one-way ANOVA, 

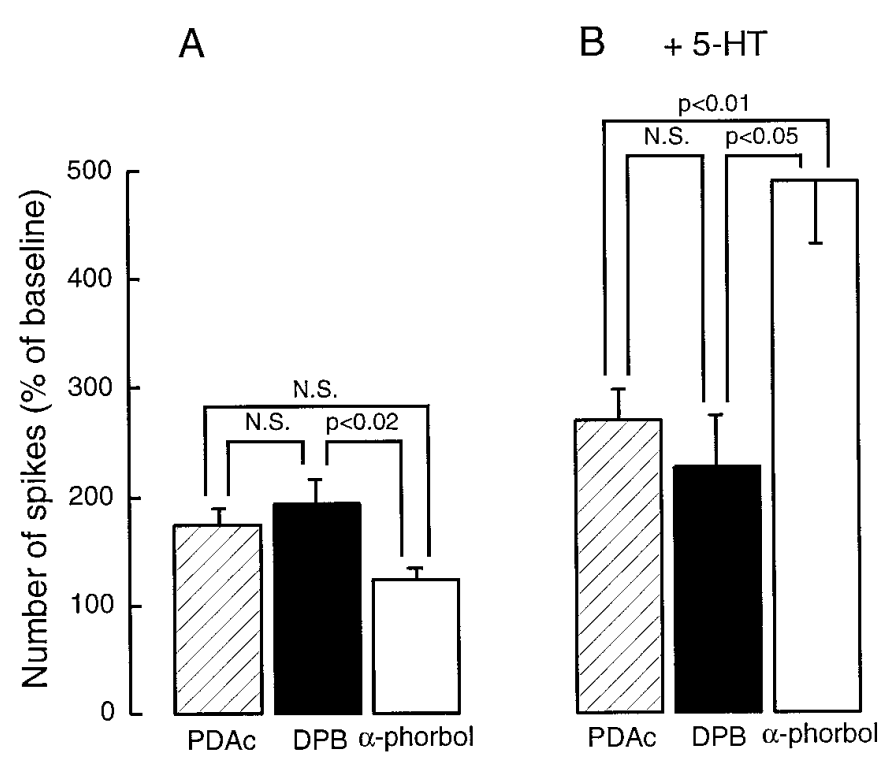

Figure 2. PKC activators induced a small increase in excitability and significantly attenuated 5-HT-induced enhancement of excitability. $A$, The summary data of the effects of PDAc $(n=7)$, DPB $(n=6)$, and $\alpha$-phorbols $(n=6)$ on excitability. At 9 min after application, PDAc and DPB caused an increase in excitability, whereas $\alpha$-phorbols had little effect on excitability. $B$, Prolonged exposure (27-30 $\mathrm{min})$ to PDAc $(n=7)$ and DPB $(n=5)$ significantly attenuated 5-HT-induced excitability, when compared with $\alpha$-phorbols. $\alpha$-Phorbols did not appear to affect 5-HTinduced enhancement of excitability (see Sugita et al., 1992).

which compared the effects of PDAc $(n=7)$, DPB $(n=5)$, and $\alpha$-phorbols $(n=6)$ on 5-HT-induced enhancement of excitability, indicated that there was a significant difference among agents $\left(F_{(2,15)}=7.03 ; p<0.01\right)$. Post hoc analysis with Tukey tests indicated that there was a significant difference between the active phorbols and inactive phorbols (PDAc versus $\alpha$-phorbol, $q_{(15,3)}=5.02, p<0.01$; DPB versus $\alpha$-phorbol, $q_{(15,3)}=4.02, p<$ $0.05)$ and that there was no significant difference between PDAc and DPB $\left(q_{(15,3)}=0.62\right)$. Thus PKC activators significantly inhibited 5-HT-induced increases in excitability, a well known cAMP-dependent effect (Klein et al., 1986; Baxter and Byrne, 1990a).

Because these experiments were conducted by using surgically isolated clusters of sensory neuron somata, it is unlikely that the observed effects on the sensory neurons by phorbol esters and 5-HT were attributable to the activation of polysynaptic pathways. Instead, the effects of phorbol esters and 5-HT were likely to be direct effects on the sensory neurons.

\section{Preincubation with activators of PKC inhibited 5-HT- induced enhancement of excitability}

We next focused on the inhibitory effects of active phorbol esters on 5-HT-induced enhancement of excitability. The inhibitory effects of active phorbols might be attributable to the deterioration of the cell by prolonged recording in the presence of activators of PKC. To exclude such possibilities, we reexamined the effects of phorbol esters under preincubation conditions. Specifically, sensory neurons were preincubated with PDAc ( $3 \mu \mathrm{M}, n=$ $10)$ or $\alpha$-phorbols ( $3 \mu \mathrm{M}, n=8)$ at least $30 \mathrm{~min}$ before implementation and recording began. Under this protocol 5-HT-induced $(10 \mu \mathrm{M})$ enhancement of excitability at 2 min after the application of 5-HT was inhibited by preincubation with PDAc when compared with $\alpha$-phorbols (PDAc, $159 \pm 18 \%$ of baseline; $\alpha$-phorbol,
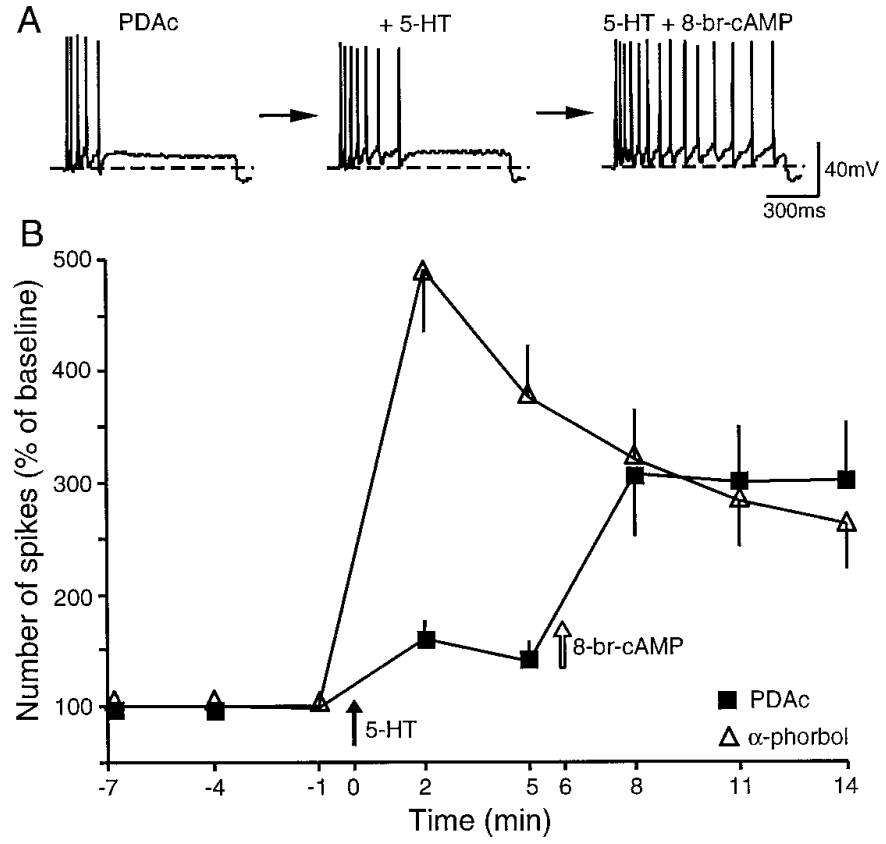

Figure 3. Prolonged preincubation in PDAc attenuated 5-HT-induced excitability. $A$, In these experiments the sensory neurons were preincubated in PDAc for 30 min before electrophysiological recordings began. Preincubation in PDAc $(n=10)$ profoundly inhibited 5-HT-induced enhancement of excitability when compared with preincubation with $\alpha$-phorbols (data not shown). The addition of 8-bromo-cAMP (5 mM) to the bath, which already contained PDAc and 5-HT, induced an additional increase in excitability. $B$, Time course of 5-HT-induced enhancement of excitability in the presence of PDAc $(n=10)$ or $\alpha$-phorbols $(n=8)$. In seven of the experiments, 8-bromo-cAMP was added to the bath, which already contained PDAc and 5-HT. The baseline excitability of the cells that were preincubated with PDAc was $3.93 \pm 0.60$. This was not significantly different from the baseline excitability of the cells preexposed to $\alpha$-phorbols $\left(3.50 \pm 0.27 ; t_{16}=0.60\right)$.

$490 \pm 54 \% ; t_{16}=6.35 ; p<0.001$ ) (Fig. 3). [The 5-HT-induced increase in excitability in the presence of $\alpha$-phorbols was very similar to that reported previously (Sugita et al., 1992)]. This result suggests that inhibitory effects of PDAc and DPB on the 5-HT-induced enhancement of excitability (Fig. 2B) were not attributable to deterioration of the cell by prolonged recording in the presence of PKC activators. Interestingly, the subsequent addition of 8-bromo-cAMP ( $5 \mathrm{mM}, n=7)$ to the bath, which already contained both 5-HT and PDAc, increased the excitability (from $138 \pm 17 \%$ to $306 \pm 70 \%$ ) (Fig. 3). Thus, the prolonged activation of PKC may inhibit 5-HT-induced enhancement of excitability at a locus upstream of cAMP.

To gain more insights into the molecular locus of inhibition, we also examined the effects of preincubation with PDAc on 8-bromo-cAMP- and $\mathrm{SCP}_{\mathrm{B}}$-induced enhancement of excitability. $\mathrm{SCP}_{\mathrm{B}}$ increases the concentration of cAMP in the sensory neurons, presumably via the interaction of $G_{\mathrm{s}}$-coupled receptors that are different from 5-HT receptors (Abrams et al., 1984; Ocorr and Byrne, 1986; Jarrard et al., 1993). The concentration of $\operatorname{SCP}_{\mathrm{B}}(20$ $\mu \mathrm{M})$ was chosen so as to induce an increase in the level of cAMP that would be comparable to that induced by $10 \mu \mathrm{M}$ 5-HT (Ocorr et al., 1985; Jarrard et al., 1993). We found that PDAc significantly inhibited both 8-bromo-cAMP-induced (5 mM) and $\mathrm{SCP}_{\mathrm{B}^{-}}$ induced enhancement of excitability. (At 11 min after cAMP application, PDAc was $253 \pm 21 \%$ of baseline; $\alpha$-phorbol was $394 \pm 45 \% ; t_{16}=2.68 ; p<0.05$. At 2 min after $\mathrm{SCP}_{\mathrm{B}}$ application, 
PDAc was $343 \pm 35 \%$ of baseline; $\alpha$-phorbol was $522 \pm 48 \%$; $t_{14}=3.02 ; p<0.01$.) Thus, prolonged activation of PKC attenuated three independent methods of activating the cAMPdependent cascade. Moreover, the results suggest that at least some of the inhibition is at a site or sites downstream of cAMP. Possible sites and mechanisms for this attenuation are presented in Discussion. The results, however, also indicated that PKC inhibited the effects of 5-HT more profoundly $(82 \%)$ than the effects of 8-bromo-cAMP $(48 \%)$ and $\mathrm{SCP}_{\mathrm{B}}(43 \%)$, suggesting specific attenuation of aspects of the 5-HT activation of the cAMP/PKA cascade.

Preincubation with activators of PKC inhibited 5-HTinduced broadening of TEA spikes but did not inhibit cAMP-induced or $\mathrm{SCP}_{\mathrm{B}}$-induced broadening

Another well characterized cAMP-mediated action of 5-HT is broadening of TEA spikes (Jarrard et al., 1993). Thus we measured the duration of action potentials in the presence of high concentrations of TEA (100 $\mathrm{mm})$ and nifedipine $(10 \mu \mathrm{M})$. High TEA blocks most potassium currents, including the delayed $\mathrm{K}^{+}$ current $\left(I_{\mathrm{K}, \mathrm{V}}\right)$ and $\mathrm{Ca}^{2+}$-dependent $\mathrm{K}^{+}$current $\left(I_{\mathrm{K}, \mathrm{Ca}}\right)$, but it does not block $I_{\mathrm{K}, \mathrm{S}}$ (Klein et al., 1982; Shuster and Siegelbaum, 1987; Baxter and Byrne, 1989). Nifedipine was used to block L-type $\mathrm{Ca}^{2+}$ currents (Edmonds et al., 1990; Braha et al., 1993). Under these conditions 5-HT-induced spike broadening is attributable primarily to the reduction of $I_{\mathrm{K}, \mathrm{S}}$ (Jarrard et al., 1993).

In preliminary experiments we found that TEA spikes were relatively unstable and that the duration of spikes gradually declined with time. This observation suggests that $\mathrm{Ca}^{2+}$ channels inactivate by repeated stimulation under the condition of prolonged spikes (Klein et al., 1980). Therefore, we examined the effects of phorbol esters, using preincubation procedures. Preincubation with PDAc $(n=11)$ for $>30$ min increased the duration of TEA spikes when compared with preincubation with $\alpha$-phorbols ( $n=9$; data not shown). This effect was statistically significant (average of three spikes was calculated in each cell; PDAc, $68.4 \pm 5.8 \mathrm{msec} ; \alpha$-phorbol, $50.7 \pm 3.9 \mathrm{msec} ; t_{17}=2.32$; $p<0.05)$. This observation is consistent with the observation that preincubation with PDAc increased excitability of the sensory neuron, possibly by a weak modulation of $I_{\mathrm{K}, \mathrm{S}}$ (Sugita et al., 1994) (also see above). Regardless of the initial duration of the spikes, we found that preincubation with PDAc strongly inhibited 5-HTinduced broadening of TEA spikes ( 1 min after 5-HT application, PDAc was $117 \pm 8 \%$ of baseline; $\alpha$-phorbol was $186 \pm 19 \%$; $t_{17}=3.70 ; p<0.01$ ) (Fig. 4). The inhibition by PDAc of 5-HTinduced broadening of TEA spikes was consistent with the attenuation by PDAc of 5-HT-induced enhancement of excitability (Figs. $2 B, 3$ ). These results support the hypothesis that activation of PKC inhibits 5-HT-induced modulation of $I_{\mathrm{K}, \mathrm{S}}$, a well known cAMP-dependent effect.

We also examined the effects of preincubation with PDAc on cAMP-induced broadening of TEA spikes. In this series of preparations as well, PDAc increased the duration of TEA spikes when compared with preincubation with $\alpha$-phorbols. Preincubation with PDAc, however, did not inhibit 8-bromo-cAMPinduced $(20 \mathrm{~mm})$ broadening of TEA spikes (3 min after cAMP application, PDAc was $217 \pm 27 \%$ of baseline; $\alpha$-phorbol was $199 \pm 28 \% ; t_{9}=0.47$ ) (Fig. 5). Therefore, the inhibitory effects of PDAc on 5-HT-induced broadening of TEA spikes appear to be at the locus upstream of cAMP. In a few experiments we examined the effects of the cAMP analog and 5-HT on the same cells. An example is illustrated in Figure $5 C$. In this neuron the effect
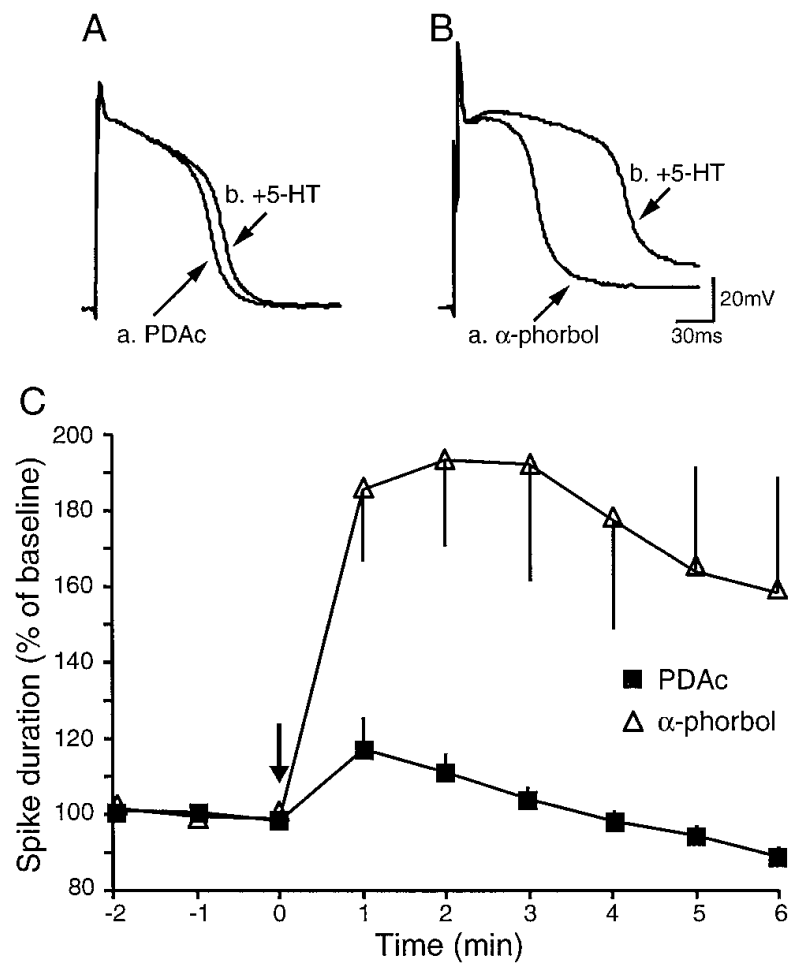

Figure 4. Prolonged preincubation in PDAc inhibited 5-HT-induced broadening of TEA spikes. The effects of PDAc on 5-HT-induced spike broadening were examined in the presence of $100 \mathrm{mM}$ TEA and $10 \mu \mathrm{M}$ nifedipine. $A$, Preincubation with PDAc $(30 \mathrm{~min})$ profoundly attenuated 5-HT-induced broadening of TEA spikes. 5-HT broadened TEA spikes by only $20 \%$ in the presence of PDAc. The traces shown were before and 1 min after application of 5-HT. B, 5-HT broadened TEA spikes by $\sim 100 \%$ in the presence of $\alpha$-phorbols. The traces shown were before and 1 min after application of 5-HT. $C$, Time course of 5-HT-induced broadening of TEA spikes in the presence of PDAc $(n=11)$ or $\alpha$-phorbols $(n=9)$.

of 5-HT was blocked almost completely by the preincubation with PDAc. The addition of 8-bromo-cAMP to the bath, which already contained 5-HT (and PDAc), broadened the spike.

$\mathrm{SCP}_{\mathrm{B}}$, like 5-HT, increases the duration of TEA spikes (Abrams et al., 1984; Pieroni and Byrne, 1992). Therefore, the effects of PDAc on $\mathrm{SCP}_{\mathrm{B}}$-induced broadening of TEA spikes were examined also. We found that PDAc did not inhibit $\mathrm{SCP}_{\mathrm{B}^{-}}$ induced broadening of the TEA spikes significantly (1 min after $\mathrm{SCP}_{\mathrm{B}}$ application, PDAc was $203 \pm 13 \%$; $\alpha$-phorbol was $235 \pm$ $16 \% ; t_{12}=1.53$ ) (Fig. 6). If we assume that $\mathrm{SCP}_{\mathrm{B}}$ and 5-HT increase the level of cAMP via a common or similar intracellular machinery but via different receptors, at least one locus of the inhibitory effects of PDAc on 5-HT-induced broadening of TEA spikes may be at the receptor level. To investigate this possibility, we examined biochemically the effects of prolonged activation of PKC by PDAc on the levels of cAMP and on 5-HT-induced increases in the level of cAMP.

\section{Prolonged incubation with PDAc increased levels of CAMP but did not attenuate 5-HT-induced increase in the level of cAMP}

The experiments were conducted in the presence of RO 20-1724 $(100 \mu \mathrm{M})$, an inhibitor of phosphodiesterase. First, we repeated previous experiments (Ocorr and Byrne, 1985; Sweatt and Kandel, 1989) that examined the ability of 5-HT to increase the cAMP content of sensory neurons involved in tail withdrawal, 
A

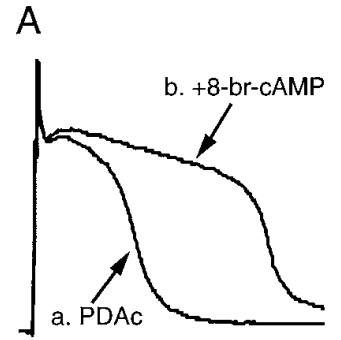

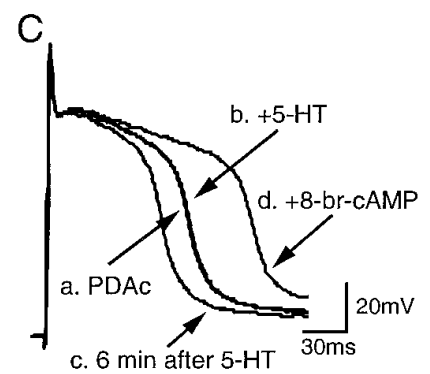

c. 6 min after $5-\mathrm{HT}$

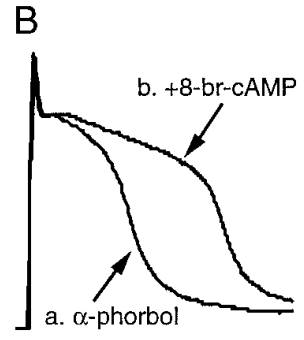

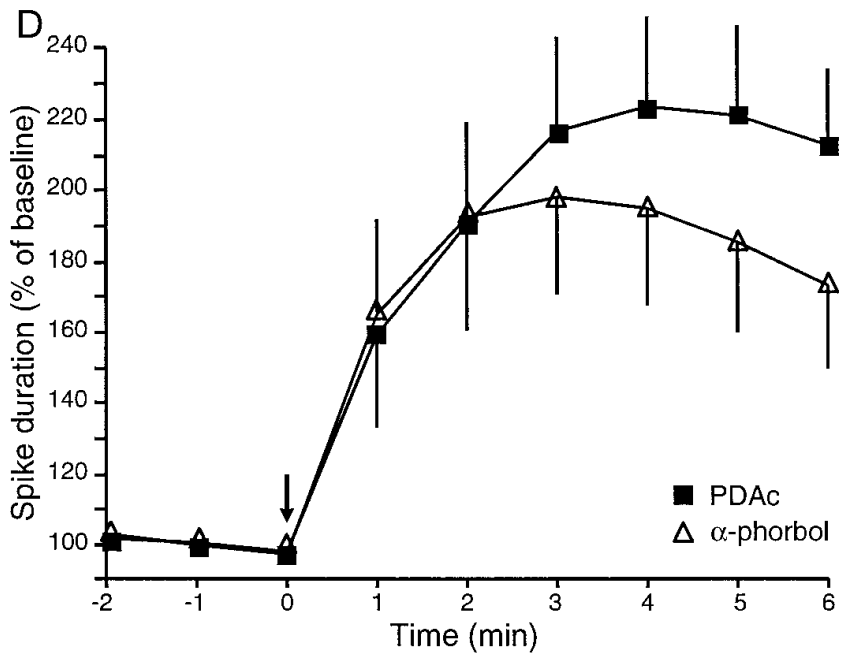

Figure 5. Preincubation in PDAc had no effect on 8-bromo-cAMP-induced broadening of TEA spikes. $A$, $B, 8$-Bromo-cAMP (20 mM) broadened TEA spikes by $\sim 100 \%$ in the presence of PDAc or $\alpha$-phorbols. The traces shown are before and 3 min after the application of 8-bromo-cAMP. Thus the inhibition of 5-HT-induced broadening of TEA spikes by PDAc (see Fig. 4) appears to be at a locus upstream of cAMP. $C, 8$-Bromo-cAMP (20 mM; 1 min after application) could broaden TEA spikes even when 5-HT failed to do so with the preincubation in PDAc. $D$, Time course of 8-bromo-cAMPinduced broadening of TEA spikes in the presence of PDAc $(n=6)$ or $\alpha$-phorbols $(n=5)$. and we confirmed their results. Higher concentrations of 5-HT were used in the biochemical experiments than in the electrophysiological ones, because the sensitivity of the methods was relatively low (see Ocorr and Byrne, 1985; Sweatt and Kandel, 1989). Bath application of 5-HT $(40 \mu \mathrm{M})$ to isolated clusters for 2 min resulted in a significant elevation of levels of cAMP (5-HT, $62.4 \pm 5.6 \mathrm{pmol} / \mathrm{mg}, n=12$; control, $38.6 \pm 2.4 \mathrm{pmol} / \mathrm{mg}, n=12$; two-tailed paired $t$ test, $\left.t_{11}=5.13 ; p<0.001\right)$.

We also examined effects of the prolonged (1 hr) activation of PKC by PDAc on the level of cAMP. Preincubation with PDAc ( 3 $\mu \mathrm{M})$ significantly increased cAMP levels, as compared with preincubation with $\alpha$-phorbols (PDAc, $43.1 \pm 6.5 \mathrm{pmol} / \mathrm{mg}, n=12$; $\alpha$-phorbols, $\left.31.2 \pm 2.8 \mathrm{pmol} / \mathrm{mg}, n=12 ; t_{11}=2.71 ; p<0.05\right)$ (Fig. 7A). Thus $\mathrm{PKC}$-induced increases in excitability may be attributable to an increase in the level of cAMP.

Finally, the effects of prolonged activation of PKC on the ability of 5-HT to stimulate synthesis of cAMP were examined. Two groups of sensory neuron clusters were examined. In one group (the 5-HT alone group), 5-HT was added to clusters that had equilibrated for $1 \mathrm{hr}$ in ASW (see Materials and Methods). In a second group (the PDAc+5-HT group), 5-HT was added to clusters that had equilibrated for $1 \mathrm{hr}$ in ASW containing $3 \mu \mathrm{M}$ PDAc. Both the 5-HT alone and PDAc+5-HT groups had matched control clusters that were equilibrated for $1 \mathrm{hr}$ in ASW or ASW plus PDAc, respectively. The matched control clusters did not receive 5-HT, however. The levels of cAMP in the 5-HT alone and PDAc+5-HT groups were expressed as percentage increases above their matched controls. In both groups application of 5-HT $(40 \mu \mathrm{M})$ for 2 min resulted in increased levels of cAMP (Fig. 7B). There was no significant difference between the two groups (5-HT alone, $162.2 \pm 12 \%$ above control, $n=12$; PDAc +5-HT, $156.9 \pm 21.9 \%$ above control, $n=12$; two-tailed unpaired $t$ test, $t_{11}=0.9$ ). Thus, prolonged activation of PKC did not attenuate 5-HT-induced increases in cAMP levels, which suggested that phorbol esters did not inhibit the actions of 5-HT at the receptor level.

\section{DISCUSSION}

\section{Interaction between PKA and PKC cascades}

Although extensive work has been conducted to elucidate second messenger/protein kinase cascades involved in serotonergic modulation of the sensory neuron of Aplysia, little is known about the interaction between these cascades. Previously, it was suggested that the cAMP/PKA cascade was necessary for the 5-HTinduced activation of the PKC pathway (Goldsmith and Abrams, 1991). Goldsmith and Abrams (1991) reported that 9-(tetrahydro2-furyl)adenine (TFHA; an inhibitor of adenylyl cyclase) inhibited 5-HT-induced facilitation of depressed synapses, an effect believed to be dependent on PKC (Braha et al., 1990; Ghirardi et al., 1992). The suggested serial interaction of $c A M P / P K A$ and $\mathrm{PKC}$, however, seems to contradict several recent findings that 5-HT-induced PKC-dependent effects are not inhibited by cAMP antagonists. For example, 5-HT-induced increases in spontaneous miniature EPSPs or 5-HT-induced increases in nifedipinesensitive $\mathrm{Ca}^{2+}$ current were not blocked by a cAMP antagonist Rp-cAMP but were blocked by inhibitors of PKC (Ghirardi et al., 1992; Braha et al., 1993). Therefore, it is questionable whether 5-HT-induced activation of PKC pathways requires the activation of PKA. Biochemical evidence that translocation of PKC is not induced by cAMP analogs also suggests that these two pathways are in parallel (Sacktor and Schwartz, 1990). Thus, the two second messenger/protein kinase cascades activated by 5-HT have been considered to be parallel or independent.

The present study raises a possibility of crosstalk between these two cascades, however. Previous studies have illustrated that a PKC cascade can interact with a $\mathrm{CAMP} / \mathrm{PKA}$ cascade at several 
A

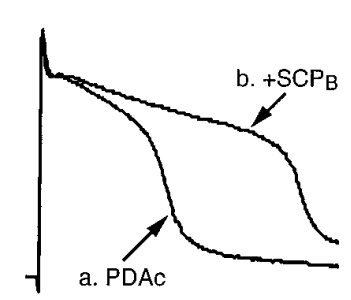

B

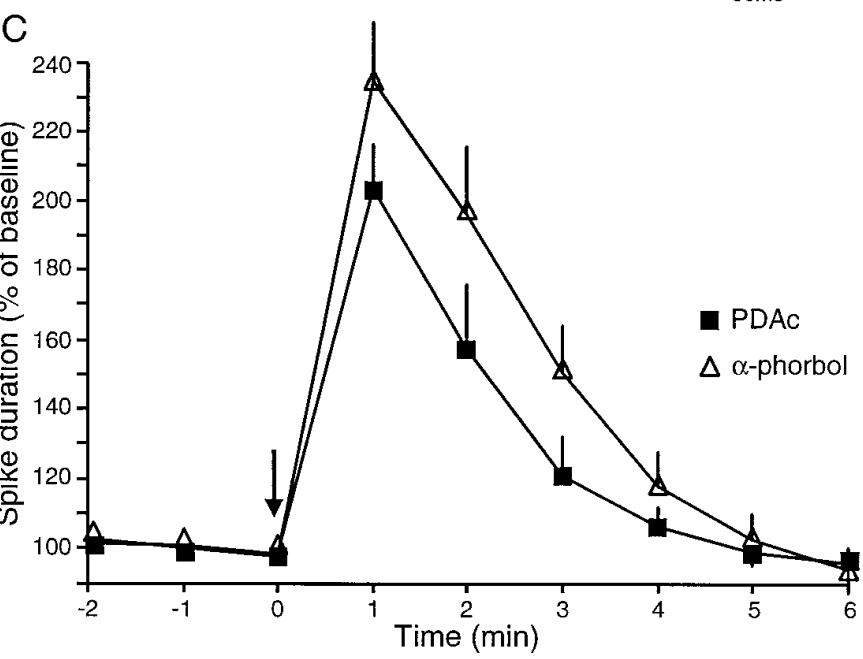

Figure 6. PDAc had no effect on $\mathrm{SCP}_{\mathrm{B}}$-induced broadening of TEA spikes. $A, B, \mathrm{SCP}_{\mathrm{B}}(20 \mu \mathrm{M})$ broadened TEA spikes by $>100 \%$ in the presence of PDAc or $\alpha$-phorbols. The traces shown are before and $1 \mathrm{~min}$ after the application of $\mathrm{SCP}_{\mathrm{B}}$. $\mathrm{SCP}_{\mathrm{B}}$-induced broadening of TEA spikes is believed to be mediated via increased levels of cAMP. Thus, the inhibition of 5-HT-induced broadening of TEA spikes by PDAc is selective. $C$, Time course of $\mathrm{SCP}_{\mathrm{B}}$-induced broadening of TEA spikes in the presence of PDAc $(n=7)$ or $\alpha$-phorbols $(n=7)$.

loci, including at the level of the adenylyl cyclase (Bell et al., 1985; Choi et al., 1993; Jacobowitz et al., 1993; Lustig et al., 1993; Yoshimura and Cooper, 1993) (for review, see Pieroni et al., 1993; Cooper et al., 1995) and at the level of the receptor (Raymond, 1991; Leidenheimer et al., 1992; Dildy-Mayfield and Harris, 1994; Raymond and Olsen, 1994; Zhang et al., 1996). Our observations support the hypothesis that one action of PKC in the sensory neuron is to elevate the level of cAMP (e.g., Fig. 7), possibly via activation of adenylyl cyclase. Incubation with PDAc and DPB, activators of PKC, caused an increase in the excitability of the sensory neuron partially mimicking a well known cAMP effect. Similar increases in excitability by either short $(5 \mathrm{~min})$ or longer ( $90 \mathrm{~min}$ ) exposure to phorbol esters recently were reported in cultured sensory neurons (Manseau et al., 1996). Moreover, preincubation with PDAc increased the level of cAMP (Fig. 7A).

We also found that PKC inhibited physiological actions of cAMP/PKA cascades, and this inhibition consists of at least two components: a component that is specific to the 5-HT-mediated activation of the cAMP/PKA pathway and a component that is common to three agents $\left(5-\mathrm{HT}\right.$, cAMP analog, $\left.\mathrm{SCP}_{\mathrm{B}}\right)$ that activate the PKA pathway. The presence of the inhibitory component specific to 5-HT was supported by our observation that preincubation with phorbol esters more profoundly attenuated 5-HTinduced increases in excitability than those induced by either 8-bromo-cAMP or $\mathrm{SCP}_{\mathrm{B}}$ and that preincubation with phorbol esters inhibited 5-HT-induced broadening of TEA spikes but had no effect on 8-bromo-cAMP- and $\mathrm{SCP}_{\mathrm{B}}$-induced broadening of
A

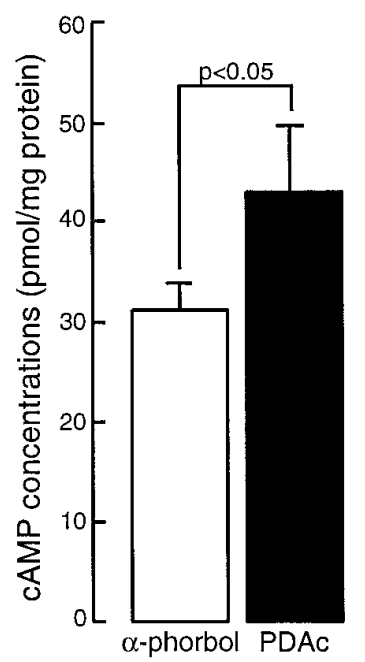

B

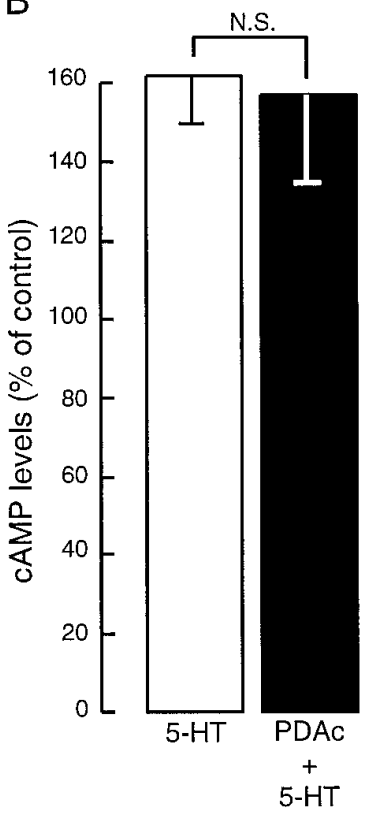

Figure 7. Measurements of levels of cAMP in the sensory neurons revealed that prolonged activation of PKC increased levels of cAMP but did not inhibit 5-HT-induced synthesis of cAMP. $A$, Preincubation in PDAc (1 hr) significantly increased cAMP levels when compared with $\alpha$-phorbols. $B$, 5-HT $(40 \mu \mathrm{M})$ increased cAMP to equivalent levels in clusters with and without incubation in PDAc $(3 \mu \mathrm{M})$.

TEA spikes. Thus PKC appeared to inhibit the effects of 5-HT specifically at a locus upstream of cAMP. If we assume that the cAMP-dependent effects of 5-HT and $\mathrm{SCP}_{\mathrm{B}}$ are mediated via common or very similar intracellular pathways but different receptors (Abrams et al., 1984; Ocorr and Byrne, 1986; Jarrard et al., 1993), a possible locus of the inhibition by PKC of 5-HTinduced effects might be at the receptor level. Our results did not support an action at the receptor for 5-HT, however. At present the locus and mechanism for PKC-induced inhibition of the 5-HT/cAMP/PKA cascade are unknown. Interestingly, it is known that the ability of 5-HT to induce cAMP-dependent effects, including enhancement of excitability and closure of $I_{\mathrm{K}, \mathrm{S}}$ and $I_{\mathrm{K}, \mathrm{Ca}}$ (Walsh and Byrne, 1989), in the sensory neuron is decreased by the continuous presence of 5-HT (Sugita et al., 1992) (also Fig. $3 B$ in this study) or repeated application of 5-HT (Walsh and Byrne, 1989). It would be interesting to examine whether PKC contributes to this phenomenon.

Preincubation with phorbol esters inhibited not only 5-HTinduced enhancement of excitability but also 8-bromo-cAMPand $\mathrm{SCP}_{\mathrm{B}}$-induced enhancement of excitability, although the inhibition of the 5-HT-induced effect is more profound. This result suggests the existence of another locus of inhibition at which PKC inhibits the enhancement of excitability by 5-HT, 8-bromo-cAMP, and $\mathrm{SCP}_{\mathrm{B}}$ via common mechanisms and that this locus lies at downstream of cAMP. One possible mechanism is the interaction between PKC- and PKA-induced modulation of ionic conductances. It is known that $I_{\mathrm{K}, \mathrm{S}}$ plays an important role in regulating excitability and that $\mathrm{CAMP} / \mathrm{PKA}$-dependent reduction of $I_{\mathrm{K}, \mathrm{S}}$ is the key component underlying 5-HT-, $\mathrm{SCP}_{\mathrm{B}^{-}}$, or 8-bromo-cAMP-induced enhancement of excitability (Klein et al., 1986; Baxter and Byrne, 1989, 1990a). Other currents, including $I_{\mathrm{K}, \mathrm{Ca}}$ and $I_{\mathrm{K}, \mathrm{V}}$, also contribute to the regulation of excitability, however (Baxter and Byrne, 1990b; Canavier et al., 1991). 
Previous work in our laboratory found that PKC increased $I_{\mathrm{K}, \mathrm{Ca}}$ (Critz and Byrne, 1992) and modulated $I_{\mathrm{K}, \mathrm{V}}$ (Sugita et al., 1994). The modulation of $I_{\mathrm{K}, \mathrm{V}}$ by $\mathrm{PKC}$ is complex and leads to a slowing of both activation and inactivation kinetics. Computer simulation studies suggested that both increases in $I_{\mathrm{K}, \mathrm{Ca}}$ and modulation of $I_{\mathrm{K}, \mathrm{V}}$ can attenuate the enhancement of excitability, which is mediated via reduction of $I_{\mathrm{K}, \mathrm{S}}$ (Baxter and Byrne, 1990b; Canavier et al., 1991). Thus, we propose that attenuation of 8-bromo-cAMP- and $\mathrm{SCP}_{\mathrm{B}^{-}}$(as well as portions of 5-HT-) induced enhancement of excitability by phorbol esters is attributable to the PKC-dependent modulation of $I_{\mathrm{K}, \mathrm{Ca}}$ and $I_{\mathrm{K}, \mathrm{V}}$. Interestingly, phorbol esters did not inhibit 8-bromo-cAMP- and $\mathrm{SCP}_{\mathrm{B}}$-induced broadening of TEA spikes (Figs. 5, 6). This lack of inhibition was likely to result from the high concentrations of TEA, which block $I_{\mathrm{K}, \mathrm{Ca}}$ and $I_{\mathrm{K}, \mathrm{V}}$ and thereby exclude the effects of PKC-dependent of modulation of these currents from the measurements.

\section{Contributions of PKC to the formation of long-term memory}

Recently, it was suggested that a prolonged (90 min) application of 5-HT, a protocol to induce long-term facilitation, led to the activation of two major subspecies of PKC in Aplysia (ApPKC I and II) (Kruger et al., 1991) for $30 \mathrm{~min}$ after treatment (Sossin et al., 1994). In addition, ApPKC I remained active for $2 \mathrm{hr}$ after treatment. Thus, prolonged activation of PKC (similar to that examined in the present study) appears to be a physiologically relevant mode of activity. Moreover, biochemical studies suggest that prolonged activation of PKC may be involved in long-term facilitatory actions of 5-HT that are mediated primarily by the PKA cascade (Scholz and Byrne, 1987, 1988; Schacher et al., 1988, 1993; Dash et al., 1990; Nazif et al., 1991; Ghirardi et al., 1995; O'Leary et al., 1995). An inhibitor of PKC, H-7, blocked 5-HT-induced long-term facilitation, whereas activators of PKC alone were not sufficient to induced long-term changes (Wu et al., 1994, 1995). The mechanisms by which the prolonged activation of PKC is involved in long-term facilitation are not clear at present, however. Our results suggest that prolonged activation of PKC by 5-HT may contribute to the maintenance of high concentrations of cAMP, although such evidence has not been obtained yet (see also Bernier et al., 1982). Additional work is necessary to test this hypothesis. In addition, it is not clear whether our observation that prolonged activation of PKC inhibits the 5-HT-induced activation of PKA pathway is related to the formation of long-term memory. Recently, the presence of a novel stage in transition between short- and long-term memory was suggested, and at this stage 5-HT-induced immediate facilitatory action is suppressed (Ghirardi et al., 1995). It would be interesting to investigate how the PKC-mediated inhibition of 5-HT-induced activation of PKA pathway is related to the suppression of 5-HT-induced immediate facilitatory action at the stage in transition between short- and long-term memory.

\section{REFERENCES}

Abrams TW, Castellucci VF, Camardo JS, Kandel ER, Lloyd PE (1984) Two endogenous neuropeptides modulate the gill and siphon withdrawal reflex in Aplysia by presynaptic facilitation involving cAMPdependent closure of a serotonin-sensitive potassium channel. Proc Natl Acad Sci USA 81:7956-7960.

Baxter DA, Byrne JH (1989) Serotonergic modulation of two potassium currents in pleural sensory neurons of Aplysia. J Neurophysiol 62:665-679.

Baxter DA, Byrne JH (1990a) Differential effects of cAMP and serotonin on membrane current, action potential duration, and excitability in somata of pleural sensory neurons of Aplysia. J Neurophysiol 64:978-990.

Baxter DA, Byrne JH (1990b) Mathematical modeling of the serotonergic modulation of electrophysiological properties of sensory neurons in Aplysia. Soc Neurosci Abstr 16:1297.

Bell JD, Dixon ILO, Brunton LL (1985) Enhancement of adenylate cyclase activity in S49 lymphoma cells by phorbol esters. Putative effects of C-kinase on the $\mathrm{G}_{\mathrm{s} \alpha}$-GTP-catalytic subunit interaction. J Biol Chem 260:2625-2628.

Bernier L, Castellucci VF, Kandel ER, Schwartz JH (1982) Facilitatory transmitter causes a selective and prolonged increase in adenosine $3^{\prime} 5^{\prime}$-monophosphate in sensory neurons mediating the gill and siphon withdrawal reflex in Aplysia. J Neurosci 2:1682-1691.

Braha O, Dale N, Hochner B, Klein M, Abrams TW, Kandel ER (1990) Second messengers involved in the two processes of presynaptic facilitation that contribute to sensitization and dishabituation in Aplysia sensory neurons. Proc Natl Acad Sci USA 87:2040-2044.

Braha O, Edmonds B, Sacktor T, Kandel ER, Klein M (1993) The contributions of protein kinase $\mathrm{A}$ and protein kinase $\mathrm{C}$ to the actions of 5-HT on L-type $\mathrm{Ca}^{2+}$ current of the sensory neurons in Aplysia. J Neurosci 13:1839-1851.

Brown RE, Javis KL, Hyland KJ (1989) Protein measurement using bicinchoninic acid: elimination of interfering substances. Anal Biochem 180:136-139.

Byrne JH (1987) Cellular analysis of associative learning. Physiol Rev 67:329-439.

Byrne JH, Kandel ER (1996) Presynaptic facilitation revisited: state and time dependence. J Neurosci 16:425-435.

Canavier CC, Baxter DA, Clark JW, Byrne JH (1991) Simulations of action potentials, transmitter release, and plasticity of sensorimotor synapses in Aplysia. Soc Neurosci Abstr 17:1590.

Carew TJ, Sahley CJ (1986) Invertebrate learning and memory: from behavior to molecules. Annu Rev Neurosci 9:435-487.

Choi E-J, Wong ST, Dittman AH, Storm DR (1993) Phorbol ester stimulation of the type I and type III adenylyl cyclases in whole cells. Biochemistry 32:1891-1894.

Cooper DMF, Mons N, Karpen JW (1995) Adenylyl cyclases and the interaction between calcium and cAMP signaling. Nature 374:421-424.

Critz SD, Byrne JH (1992) Modulation of $I_{\mathrm{K}, \mathrm{Ca}}$ by phorbol estermediated activation of PKC in pleural sensory neurons of Aplysia. J Neurophysiol 68:1079-1086.

Dash PK, Hochner B, Kandel ER (1990) Injection of the cAMPresponsive element into the nucleus of Aplysia sensory neurons blocks long-term facilitation. Nature 345:718-721.

Dildy-Mayfield JE, Harris RA (1994) Activation of protein kinase C inhibits kainate-induced currents in oocyte expressing glutamate receptor subunits. J Neurochem 62:1639-1642.

Edmonds B, Klein M, Dale N, Kandel ER (1990) Contribution of two types of calcium channels in synaptic transmission and plasticity. Science 250:1142-1147.

Ghirardi M, Braha O, Hochner B, Montarolo PG, Kandel ER, Dale N (1992) Roles of PKA and PKC in facilitation of evoked and spontaneous transmitter release at depressed and nondepressed synapses in Aplysia sensory neurons. Neuron 9:479-489.

Ghirardi M, Montarolo PG, Kandel ER (1995) A novel intermediate stage in the transition between short- and long-term facilitation in the sensory to motor synapse of Aplysia. Neuron 14:413-420.

Goldsmith BA, Abrams TW (1991) Reversal of synaptic depression by serotonin at Aplysia sensory neuron synapses involves activation of adenylate cyclase. Proc Natl Acad Sci USA 88:9021-9025.

Hawkins RD, Kandel ER, Siegelbaum SA (1993) Learning to modulate transmitter release: themes and variations in synaptic plasticity. Annu Rev Neurosci 16:625-665.

Huganir RL, Greengard P (1990) Regulation of neurotransmitter receptor desensitization by protein phosphorylation. Neuron 5:555-567.

Jacobowitz O, Chen J, Premont RT, Iyengar R (1993) Stimulation of specific type of $\mathrm{G}_{\mathrm{s}}$-stimulated adenylyl cyclases by phorbol ester treatment. J Biol Chem 268:3829-3832.

Jarrard HE, Goldsmith BA, Abrams TW (1993) In Aplysia sensory neurons, the neuropeptide $\mathrm{SCP}_{\mathrm{B}}$ and serotonin differ in efficacy both in modulating cellular properties and in activating adenylyl cyclase: implications for mechanisms underlying presynaptic facilitation. Brain Res 616:188-199.

Kandel ER, Schwartz JH (1982) Molecular biology of learning: modulation of transmitter release. Science 218:433-443. 
Klein M, Shapiro E, Kandel ER (1980) Synaptic plasticity and modulation of the calcium current. J Exp Biol 89:117-157.

Klein M, Camardo J, Kandel ER (1982) Serotonin modulates a specific potassium current in the sensory neurons that show presynaptic facilitation in Aplysia. Proc Natl Acad Sci USA 79:5713-5717.

Klein M, Hochner B, Kandel ER (1986) Facilitatory transmitters and cAMP can modulate accommodation as well as transmitter release in Aplysia sensory neurons: evidence for parallel processing in a single cell. Proc Natl Acad Sci USA 83:7994-7998.

Kruger KE, Sossin WS, Sacktor TC, Bergold PJ, Beushausen S, Schwartz $\mathrm{JH}$ (1991) Cloning and characterization of $\mathrm{Ca}^{2+}$-dependent and $\mathrm{Ca}^{2+}$-independent PKCs expressed in Aplysia sensory cells. J Neurosci 11:2303-2313.

Leidenheimer NJ, McQuilkin SJ, Hahner LD, Whiting P, Harris RA (1992) Activation of protein kinase C selectively inhibits the gammaaminobutyric acid A receptor: role of desensitization. Mol Pharmacol 41:1116-1123.

Lustig KD, Conklin BR, Herzmark P, Taussig R, Bourne HR (1993) Type II adenylyl cyclase integrates coincident signals from $G_{s}, G_{i}$, and $\mathrm{G}_{\mathrm{q}}$. J Biol Chem 268:13900-139005.

Manseau F, Sossin WS, Castellucci VF (1996) Role of PKC in intermediate forms of memory; modulation of synaptic transmission and excitability. Soc Neurosci Abstr 22:695.

Nazif FA, Byrne JH, Cleary LJ (1991) cAMP induces long-term morphological changes in sensory neurons of Aplysia. Brain Res 539:324-327.

Ocorr KA, Byrne JH (1985) Membrane responses and changes in cAMP levels in Aplysia sensory neurons by serotonin, tryptamine, FMRFamide, and small cardioactive peptide $\mathrm{B}\left(\mathrm{SCP}_{\mathrm{B}}\right)$. Neurosci Lett 55:113-118.

Ocorr KA, Byrne JH (1986) Evidence for separate receptors that mediate parallel effects of serotonin and small cardioactive peptide B $\left(\mathrm{SCP}_{\mathrm{B}}\right)$ on adenylate cyclase in Aplysia Californica. Neurosci Lett 70:283-288

Ocorr KA, Walters ET, Byrne JH (1986) Associative conditioning analog selectively increases cAMP levels of tail sensory neurons in Aplysia. Proc Natl Acad Sci USA 82:2548-2552.

O’Leary FA, Byrne JH, Cleary LJ (1995) Long-term structural remodeling in Aplysia sensory neurons requires de novo protein synthesis during a critical time period. J Neurosci 15:3519-3525.

Pieroni JP, Byrne JH (1992) Differential effects of serotonin, FMRFamide, and small cardioactive peptide on multiple distributed processes modulating sensorimotor synaptic transmission in Aplysia. J Neurosci 12:2633-2647.

Pieroni JP, Jacobowitz O, Chen J, Iyengar R (1993) Signal recognition and interaction by $\mathrm{G}_{\mathrm{s}}$-stimulated adenylyl cyclase. Curr Opin Neurobiol 3:345-351.

Raymond JR (1991) Protein kinase C induces phosphorylation and desensitization of the human 5-HT1A receptor. J Biol Chem 266:14747-14753.

Raymond JR, Olsen CL (1994) Protein kinase A induces phosphorylation of the human 5-HT1A receptor and augments its desensitization by protein kinase C in CHO-K1 cells. Biochemistry 33:11264-11269.
Sacktor TC, Schwartz JH (1990) Sensitizing stimuli cause translocation of protein kinase C in Aplysia sensory neurons. Proc Natl Acad Sci USA 87:2036-2039.

Schacher S, Castellucci VF, Kandel ER (1988) cAMP evokes long-term facilitation in Aplysia sensory neurons that requires new protein synthesis. Science 240:1667-1669.

Schacher S, Kandel ER, Montarolo PG (1993) cAMP and arachidonic acid stimulate long-term structural and functional changes produced by neurotransmitters in Aplysia sensory neurons. Neuron 10:1079-1088.

Scholz KP, Byrne JH (1987) Long-term sensitization in Aplysia: biophysical correlates in tail-sensory neurons. Science 235:685-687.

Scholz KP, Byrne JH (1988) Intracellular injection of cAMP induces a long-term reduction of neuronal $\mathrm{K}^{+}$currents. Science 240:1664-1666.

Shuster MJ, Siegelbaum SA (1987) Pharmacological characterization of serotonin-sensitive potassium channel of Aplysia sensory neurons. J Gen Physiol 90:587-608.

Sossin WS, Sacktor TC, Schwartz JH (1994) Persistent activation of protein kinase $\mathrm{C}$ during the development of long-term facilitation in Aplysia. Learning Memory 1:189-202.

Sugita S, Goldsmith JR, Baxter DA, Byrne JH (1992) Involvement of protein kinase $\mathrm{C}$ in serotonin-induced spike broadening and synaptic facilitation of sensorimotor connections in Aplysia. J Neurophysiol 68:643-651.

Sugita S, Baxter DA, Byrne JH (1994) Activation of protein kinase C mimics serotonin-induced modulation of a voltage-dependent potassium current in pleural sensory neurons of Aplysia. J Neurophysiol 72:1240-1249.

Sugita S, Baxter DA, Byrne JH (1997) Differential effects of 4-aminopyridine, serotonin, and phorbol esters on facilitation of sensorimotor connections in Aplysia. J Neurophysiol 77:177-185.

Sweatt JD, Kandel ER (1989) Persistent and transcriptionally dependent increase in protein phosphorylation in long-term facilitation of Aplysia sensory neurons. Nature 339:51-54.

Walsh JP, Byrne JH (1989) Modulation of a steady-state $\mathrm{Ca}^{2+}$-activated $\mathrm{K}^{+}$current in tail sensory neurons of Aplysia: role of serotonin and cAMP. J Neurophysiol 61:32-44.

Wu F, Friedman L, Schacher S (1994) Increase in PKA or PKC activity regulates neuronal outgrowth by Aplysia sensory neurons in culture. Soc Neurosci Abstr 20:816.

Wu F, Friedman L, Schacher S (1995) Transient versus persistent functional and structural changes associated with facilitation of Aplysia sensorimotor synapses are second messenger-dependent. J Neurosci 15:7517-7527.

Yanow SK, Sossin WS (1996) Regulation of translation by PKC and PKA during intermediate phases of facilitation of Aplysia. Soc Neurosci Abstr 22:696.

Yoshimura M, Cooper DMF (1993) Type-specific stimulation of adenylyl cyclase by protein kinase C. J Biol Chem 268:4604-4607.

Zhang L, Yu Y, Mackin S, Weight FF, Uhl GR, Wang JB (1996) Differential $\mu$ opiate receptor phosphorylation and desensitization induced by agonists and phorbol esters. J Biol Chem 271:11449-11454. 